\title{
Ethynylbenzene Monolayers on Gold: A metal-molecule binding motif derived from a hydrocarbon.
}

\author{
Andrew M. McDonagh,$^{\dagger^{*}}$ M. Hadi Zareie, ${ }^{\dagger *}$ Michael J. Ford,$^{\dagger}$ Chris Barton, ${ }^{\ddagger}$ Milena Ginic-Markovic, ${ }^{\S}$ \\ Janis G. Matisons ${ }^{\S}$ \\ ${ }^{\dagger}$ Institute for Nanoscale Technology, University of Technology Sydney, Broadway, \\ New South Wales, 2007, Australia, \\ ${ }^{+}$CSIRO Industrial Physics, P.O. Box 218, Lindfield, NSW 2070, Australia \\ ${ }^{\S}$ School of Chemistry Physics and Earth Science, The Flinders University of South Australia, \\ GPO Box 2100, SA 5001, Australia.
}

andrew.mcdonagh@uts.edu.au

hadi.zareie@uts.edu.au

RECEIVED DATE (to be automatically inserted after your manuscript is accepted if required according to the journal that you are submitting your paper to)

Ethynylbenzene monolayers on gold

\section{Abstract}

Exposure of a $\mathrm{Au}(111)$ surface to ethynylbenzene in solution leads to the formation of a bound hydrocarbon monolayer. A chemisorption process occurs to give a stable layer consisting of oxygencontaining hydrocarbon species. Ethynylbenzene itself does not oxidize under the deposition conditions 
indicating that the gold surface facilitates the oxidation process. Calculations show that ethynylbenzene and its oxidation products phenylacetic acid and phenyloxirane have positive binding energies to the gold surface. 1,4-diethynylbenzene also binds to $\mathrm{Au}(111)$ and anchors gold nanoparticles deposited from solution to form dense, semi-regular arrays. 


\section{Introduction}

Self-assembled monolayers (SAMs) of molecules on gold surfaces have typically used thiols to anchor compounds of interest to the surface. ${ }^{1}$ Other groups such as pyridines, ${ }^{2}$ nitriles, ${ }^{3}$ and isocyanides ${ }^{4,5}$ have also been utilized as anchors to gold. In the design of molecular wires, the phenylethynyl moiety features strongly ${ }^{6}$ due to its highly delocalized electronic structure. Molecular wires containing this group almost exclusively use thiols as the molecular "alligator clips" when attached to a gold surface.

Given that gold(I) phenylacetylide complexes can be readily prepared, ${ }^{7}$ we sought to investigate if the alkynyl fragment could be used directly as an anchoring group to form SAMs on gold. Previous studies employing surface-enhanced Raman spectroscopy (SERS) showed that ethynylbenzene (Figure 1) binds to gold surfaces ${ }^{8}$ and nanoparticles. ${ }^{9}$ Feilchenfeld and Weaver $^{8(a)}$ deduced from SERS data that ethynylbenzene attaches to gold surfaces via the $\mathrm{C} \equiv \mathrm{C}$ moiety. They suggested that the ethynyl group is oriented horizontally relative to the metal surface. Joo and $\mathrm{Kim}^{9}$ provide SERS data to suggest that the phenyl ring adopts a vertical orientation when ethynylbenzene is bound to gold nanoparticles but do not offer a description of the overall packing formation. We have recently presented density functional calculations showing that the terminal carbon of ethynylbenzene can make a strong bond with the $\mathrm{Au}(111)$ surface and that a perpendicular orientation of the molecule to the surface is preferred. ${ }^{10}$
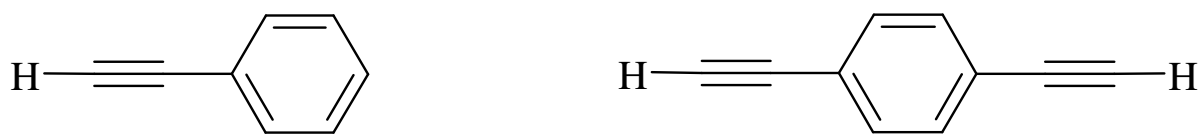

Figure 1. Compounds used in this work, ethynylbenzene (left) and 1,4-diethynylbenzene (right).

However, recent work by Deng and Friend ${ }^{11}$ using a similar compound, styrene, has demonstrated the ability of extended planar gold surfaces dosed with atomic oxygen to effectively oxidize the bound organic molecules to form epoxides and carboxylic acids. We therefore anticipated that monolayer formations anchored to gold via alkyne groups may also be susceptible to oxidation. Pritzkow and Rao ${ }^{12}$ showed that ethynylbenzene is oxidized by $\mathrm{O}_{2}$ at $110{ }^{\circ} \mathrm{C}$, with benzoic acid, benzoic anhydride, and 
benzaldehyde the main oxidation products. Thermal dimerization, oligomerization, and polymerization processes were also observed. $^{12,13}$

In this work, we present data showing that ethynylbenzene forms monolayer structures on Au(111) surfaces by solution-phase deposition and that the deposited monolayer is comprised of a bound, oxygen-containing species.

\section{Results and Discussion}

Surface Layers on $\mathrm{Au}(\mathbf{1 1 1}) . \mathrm{Au}(111)$ substrates were immersed in $1 \mathrm{mM}$ ethanolic solutions of ethynylbenzene containing ammonium hydroxide. The samples were incubated at $60^{\circ} \mathrm{C}$ for 24 hours before being thoroughly rinsed with solvent and dried with nitrogen gas. The addition of base was found to be essential for monolayer formation; no structures were observed (by scanning tunneling microscopy) when base was omitted. We have previously reported a discussion of possible mechanisms for the initial monolayer formation. ${ }^{10}$

Ellipsometry and contact angle. The thickness of the deposited layer on gold was measured by ellipsometry. A thickness of $0.6 \pm 0.1 \mathrm{~nm}$ was obtained using a refractive index for the organic layer of 1.55. The measured thickness is less than the calculated thickness of $0.8 \mathrm{~nm}$ for an ethynylbenzene monolayer in a perpendicular orientation relative to the surface, ${ }^{10}$ suggesting that the molecules are not perpendicular with respect to the gold surface. This is also supported by analysis of images obtained by scanning tunneling microscopy (see below).

Water contact angles were measured on freshly prepared surfaces. An advancing angle of $70^{\circ}$ was recorded for the ethynylbenzene treated sample while an angle of $62^{\circ}$ was recorded for the untreated gold substrate under the same experimental conditions. The advancing contact angle for the deposited layer closely matches the value of $70^{\circ}$ reported for a SAM of benzenethiol on gold, ${ }^{14}$ which should have a similar thickness and presents a similar aromatic structure to the contacting liquid. 


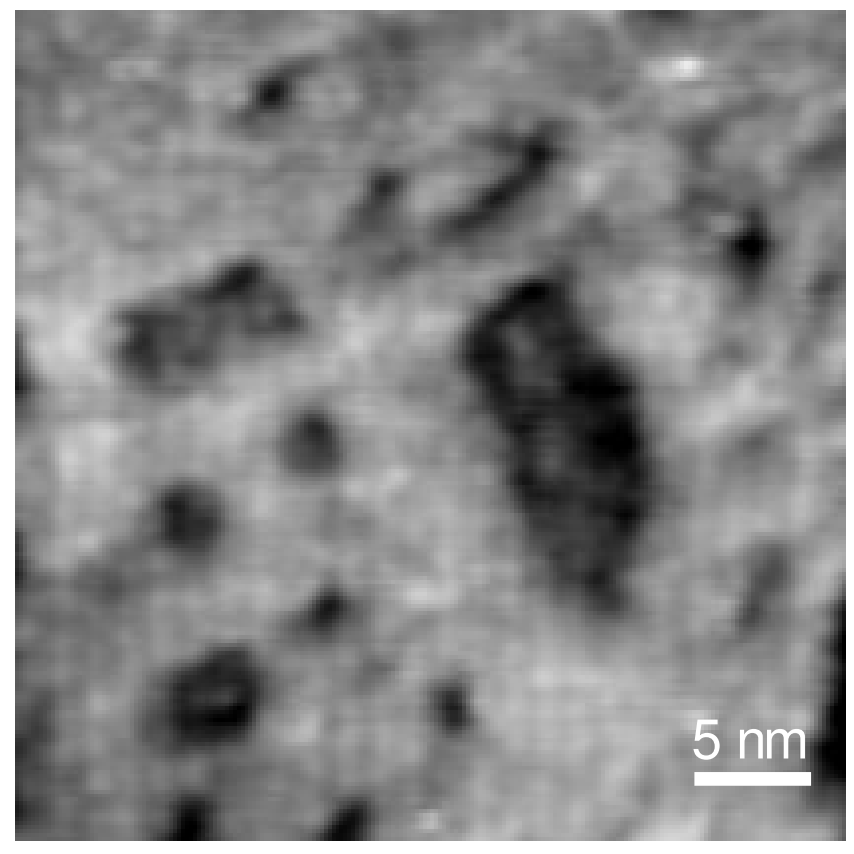

Figure 2. STM image of a monolayer formed by exposure of a $\mathrm{Au}(111)$ surface to an ethynylbenzene solution.

Scanning Tunneling Microscopy. The structures of assembled films were studied using scanning tunneling microscopy (STM). A representative large area STM image is shown in Figure 2. The surface structure is significantly different to that of bare gold. Features such as depressions and domain boundaries are visible. We deduce that the depressions (shown as dark regions) are vacancy islands that are concomitant with a rearrangement of the $\mathrm{Au}$ surface atoms during the chemisorption of ethynylbenzene molecules. ${ }^{15}$ The measured depth of these vacancy islands is $0.24 \pm 0.02 \mathrm{~nm}$, in agreement with the interplane distance of $0.236 \mathrm{~nm}$ in the fcc Au crystal along the (111) direction. ${ }^{16} \mathrm{~A}$ mechanism for the formation of these pits upon molecular adsorption has been proposed by Poirier ${ }^{17}$ and refined by Yang and Liu. ${ }^{18}$ The basis of this mechanism is that the bare reconstructed Au(111) relaxes back to the bulk terminated surface upon adsorption. The surface layer atoms are more densely packed, by about $4.4 \%$, in the reconstructed surface and so gold adatoms are created on the surface upon molecular adsorption. Subsequent growth and organization of the overlayer can then lead to vacancy and adatom islands. The removal of surface layer gold atoms by the adsorbed molecule returning to solution could also contribute to pit formation. Also, evident in Figure 2 are features corresponding to individual 
molecular units. These features measure $\sim 0.9 \mathrm{~nm}$ in diameter. This distance is larger than the $0.5 \mathrm{~nm}$ diameter of a phenyl unit, which would be the expected feature size for a molecule oriented in a perpendicular fashion to the surface. This suggests that the molecules adopt a more horizontal orientation relative on to the surface. The STM images contain some regions of short-range molecular ordering although no long-range ordering is apparent.

To ascertain if any of the observed surface structures were due only to the solvent/hydroxide mixture used in the deposition phase of the experiment, STM experiments were conducted on a bare gold surface subjected to the same conditions as the surface layer shown in Figure 2 except that ethynylbenzene was not added. In this case, no structures other than that of $\mathrm{Au}(111)$ were detected.

We conclude from the STM data that the ethynylbenzene is chemically bound to the $\mathrm{Au}(111)$ surface as a monolayer with only a minor degree of organization.

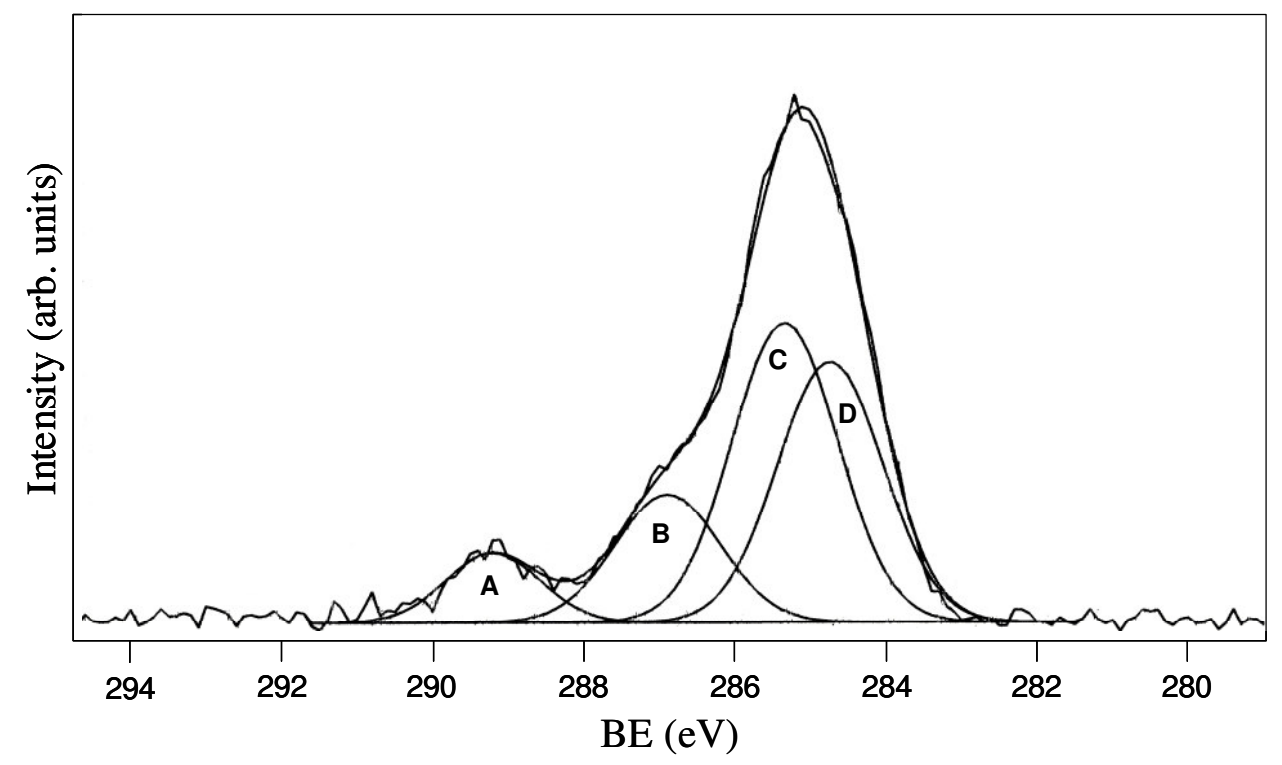

Figure 3. C 1s XPS spectrum of a monolayer on $\operatorname{Au}(111)$ showing curves fitted to the experimental data. The labels A-D indicate the fitted components. The atom ratios A:B:C:D are $1: 1.8: 5.4: 4.7$.

X-ray photoelectron spectroscopy (XPS). XPS spectra were recorded of freshly prepared samples of the molecular structures on $\mathrm{Au}(111)$, aged molecular structures on $\mathrm{Au}(111)$, and $\mathrm{Au}(111)$ substrates after exposure to an ammonium hydroxide/ethanol solution in the absence of ethynylbenzene. 
Figure 3 shows the $\mathrm{C}$ 1s spectral region obtained from a freshly prepared sample. The fitted curves (AD) show that the spectrum consists of four peaks with binding energies of $284.7,285.3,286.9$ and $289.2 \mathrm{eV}$. Previous XPS experiments ${ }^{19}$ investigating ethynylbenzene bound to $\mathrm{Cu}(100)$ showed $\mathrm{C} 1 \mathrm{~s}$ signals at $283.2 \mathrm{eV}$, assigned to the two acetylenic carbon atoms bound to the metal, together with signals at 284.2, 284.5 and $285.2 \mathrm{eV}$ assigned to the carbon atoms of the aromatic ring. In the current work, the signals at $285.3 \mathrm{eV}$ (C) and $284.7 \mathrm{eV}$ (D) may be assigned to the carbon atoms of the aromatic ring by comparison. However, the signal at $289.2 \mathrm{eV}(\mathrm{A})$ is characteristic of a carbon atom in a carboxylic acid group. ${ }^{20}$ Assignment of the peak at $286.9 \mathrm{eV}$ (B) is more speculative although it is similar in binding energy to the value of $286.6 \mathrm{eV}$ reported for epoxide carbon atoms in phenyloxirane (styrene oxide) bound to $\operatorname{Ag}(111) .^{21}$

The acetylenic carbons of ethynylbenzene shift to lower binding energies of $\sim 283.2 \mathrm{eV}$ when attached to $\mathrm{Cu}(100)^{19}$ and $\mathrm{Rh}(100)^{22}$ surfaces. A di- $\sigma$ bond between the alkynyl carbon atoms and the metal surface is proposed as the likely bonding mode. In the current work, this mode of bonding is not indicated due to the absence of peaks below $284 \mathrm{eV}$. Inspection of the reported XPS spectra for styrene films on silver surfaces, ${ }^{21,23}$ reveals no peaks below $284 \mathrm{eV}$. DFT calculations indicated ${ }^{21}$ that the signal assigned to the metal-bound carbon atom is located at a binding energy that overlaps with those of most of the aromatic carbon atoms. The current data are consistent with a similar binding mode. Although we are unable to unambiguously discern the identity of the molecular species, the data are indicative of a mixture of oxidized aromatic hydrocarbon species, such as phenylacetic acid and phenyloxirane. However, we cannot rule the possibility of a surface-bound oxametallocycle, which has been suggested as an intermediate structure in the oxidation of styrene on $\mathrm{Au}(111){ }^{11}$.

The XPS spectrum also contains signals in the O 1s region. Deconvolution of the experimental data (see Supporting Information) yields three peaks with binding energies of 533.6, 532.6 and $531.2 \mathrm{eV}$. These data are consistent with the above conclusions and suggest that there is more than one oxidized species on the surface (for example, phenylacetic acid alone should give only two $\mathrm{O}$ 1s signals and phenyloxirane only one). 
No signals due to any nitrogen species, which may arise from the ammonium hydroxide, were observed suggesting that it does not bind to the gold surface, nor react to form nitrogen-containing organic compounds that bind to the gold.

An XPS spectrum of the same sample was recorded after one week of exposure to an ambient atmosphere (away from light). The spectrum is virtually identical in all respects indicating that the monolayer is stable over this period of time. This result also suggests that oxidation of ethynylbenzene occurs during the deposition phase.

XPS experiments were performed using gold substrates immersed in solution at $60{ }^{\circ} \mathrm{C}$ for 24 hours without ethynylbenzene to ascertain if the spectra discussed above result from exposure of the gold surface to the ethanol/ammonium hydroxide solution. The C 1s spectrum (see Supporting Information) is significantly different to that of the sample prepared using ethynylbenzene. Peaks for carboncontaining compounds are seen at 289.1, 287.1 and $285.2 \mathrm{eV}$, which is similar to the sample prepared using ethynylbenzene, however the observed atom ratios of $1: 1.4: 18.1$, respectively, are markedly different. Furthermore, significantly more carbon was deposited compared to the sample prepared using ethynylbenzene. Using the reduction in intensity of the $\mathrm{Au} 4 \mathrm{f}$ signal to gauge the relative layer thicknesses, a total carbon to gold atom ratio of $2.4: 1$ was recorded for this sample compared to 0.78 : 1 for the ethynylbenzene-containing specimen. This behaviour has been documented previously where XPS indicated that carbon- and oxygen-containing contaminants formed thicker layers than SAMs of long-chain alkanethiol molecules on gold surfaces. ${ }^{24}$ It was argued that the formation of thiolate SAMs lowers the free surface energy. Thus, less contaminant overlayers are physisorbed on the modified gold surface compared to an unmodified surface.

The $\mathrm{O} 1 \mathrm{~s}$ region of the spectrum is also different to that of the ethynylbenzene sample. Signals at 533.5 and $532.3 \mathrm{eV}$ are observed while no signal at $531.2 \mathrm{eV}$ is apparent. The carbon to oxygen ratio is also significantly higher in these specimens. Another significant difference is a peak in the $\mathrm{N} 1 \mathrm{~s}$ region of the spectrum. These results indicate that the XPS spectra obtained with ethynylbenzene present are not simply the result of the ethanol/ammonium hydroxide solution. 
We conclude from the XPS data that the monolayer formed from ethynylbenzene contains carbon and oxygen only, and that the bound organic molecules are probably oxidized derivatives of ethynylbenzene. Furthermore, no monolayer degradation occurs after exposure to air for one week and the layer is significantly different to that deposited on gold from the solvent/base mixture.

Infra-red Spectroscopy. Polarization modulation infra-red reflection absorption spectroscopy (PMIRRAS) was used to further characterize the films on gold surfaces. Data in the fingerprint region, 1200$1700 \mathrm{~cm}^{-1}$ was obtained (see Supporting Information) however outside this region, the signal-to-noise ratio was such that no distinct bands could be reliably identified. Bands consistent with carboxylate groups are observed at 1397 and $1607 \mathrm{~cm}^{-1}$ while bands consistent with aromatic ring stretching $\operatorname{modes}^{25}$ are evident at 1512,1607 and $1652 \mathrm{~cm}^{-1}$. We are more cautious with assignment of the band at $1250 \mathrm{~cm}^{-1}$. However a band at similar wavenumber has been reported ${ }^{26}$ and assigned to ether groups. Although the PM-IRRAS data does not enable unambiguous identification, the data are consistent with oxygen-containing organic species bound to the gold surface.

${ }^{1}$ H NMR Experiments. As mentioned previously, the oxidation of ethynylbenzene by $\mathrm{O}_{2}$ has been reported as well as the oxidation of styrene catalyzed by planar gold surfaces. ${ }^{1} \mathrm{H}$ NMR experiments were performed to ascertain if the oxidation products detected by XPS result simply from the preparation conditions. A $1 \mathrm{mM}$ solution of ethynylbenzene in deuterated ethanol (ethanol- $\mathrm{d}_{6}$ ) was prepared and subjected to the same conditions used for the surface layer deposition, i.e. $60{ }^{\circ} \mathrm{C}$ with ammonium hydroxide but with no gold surface present. ${ }^{1} \mathrm{H}$ NMR spectra were recorded before addition of ammonium hydroxide and heating, after addition of ammonium hydroxide but before heating, and after heating the basic solution at $60{ }^{\circ} \mathrm{C}$ (see Supporting Information). The spectrum of ethynylbenzene in ethanol shows the expected signals at $\delta$ 7.41-7.44 assigned to two protons of the aromatic ring, signals at $\delta 7.28-7.33$ assigned to the remaining three protons of the aromatic ring, and a distinctive signal at $\delta 3.39$ assigned to the acetylenic proton. Upon addition of ammonium hydroxide (2 molar equivalents) to the solution, no change in these signals is observed. Upon heating the solution at $60{ }^{\circ} \mathrm{C}$ the signal assigned to the acetylenic proton diminishes and after 42 hours it has almost disappeared. The 
aromatic proton signals, in contrast, remain unchanged. Importantly, no new signals are detected in the spectrum during the course of the experiment. Of the possible processes that could occur during this experiment, we rule out polymerization reactions as these would give rise to new resonances in the ${ }^{1} \mathrm{H}$ spectrum, and likewise oxidation reactions leading to products such as benzoic acid, phenylacetic acid or phenyloxirane would also give rise to new signals. We conclude that under these conditions, ethynylbenzene has simply been deprotonated and the proton exchanged for deuterium from the solvent, as shown in Scheme 1.

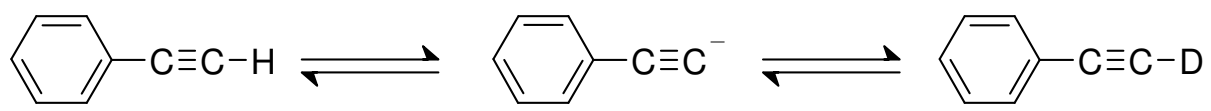

Scheme 1. Proposed reaction of ethynylbenzene with ammonium hydroxide in ethanol- $\mathrm{d}_{6}$. The equilibrium lies far to the right due to the large excess of deuterated solvent.

Although we cannot rule out any formation of the homocoupled product 1,4-diphenylbutadiyne, which has a virtually identical ${ }^{1} \mathrm{H}$ NMR spectrum to ethynylbenzene minus the acetylenic proton signal, it is clear that no oxidized species containing carboxylic acid or epoxide groups (as indicated by XPS) are present. The formation of these groups in the surface-bound structures must therefore involve the gold substrate.

Computational Studies. To shed further light on the experimental results described here, we calculated the adsorption energetics for the oxygen-containing species phenylacetic acid and phenyloxirane (Figure 5). These compounds have been detected as oxidation products of styrene catalyzed by planar gold surfaces that have been prepared by dosing with atomic oxygen. ${ }^{11}$ The calculations employ the SIESTA $^{27,28}$ software package, which implements density functional theory within the linear combination of atomic orbitals approximation. Further details of the calculations are provided in the supporting information. Convergance of the calculated interaction energies as a function of the computational parameters has previously been tested by us in detail. ${ }^{10}$ The interactions reported here are converged to better than $7 \mathrm{kcal} \mathrm{mol}^{-1}$, not including basis set effects. 

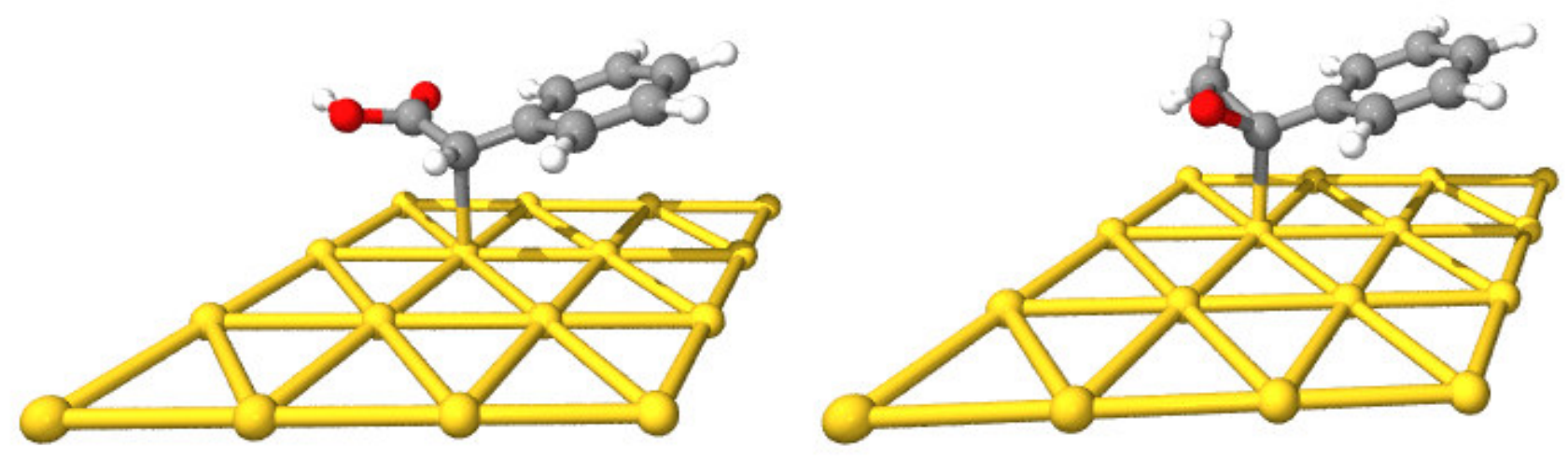

Figure 5. Phenylacetic acid (left), and phenyloxirane (right) on $\mathrm{Au}(111)$ surfaces.

The molecules were first geometry optimized in the gas phase and then the adsorbed molecule was reoptimized on the gold surface starting from the atop and hollow sites. The gold slab was held fixed at its optimum bulk geometry, that is, surface relaxation effects were not considered. This is a relatively small effect that is below the overall convergance level of the calculations with respect to the computational parameters. A mixed z-matrix / Cartesian coordinate format was used to define the structures and perform the optimizations. ${ }^{29}$ In both cases, the hollow site is unbound and results in the molecules moving away from the surface. The atop sites minimize to the structure shown in Figure 5. At the optimized geometry, the metal-bound carbon atom is $2.36 \AA$ and $2.22 \AA$ above the gold atom in the phenylacetic acid and phenyloxirane, respectively. The binding energies of the molecules to the surface were calculated using the standard counterpoise correction technique of Boys and Bernardi. ${ }^{30}$ The counterpoise method is widely used to correct for basis set superposition errors (BSSE) and is appropriate for modest size basis-sets such as the double-zeta plus polarization used in the present work. We would expect the BSSE to be about $10 \% .{ }^{10}$ The binding energies are relative to the isolated gold slab and radical in the adsorbed geometry, i.e. they are vertical, or non-adiabatic binding energies. The calculated values are $13.5 \mathrm{kcal} \mathrm{mol}^{-1}$ for phenylacetic acid and $20 \mathrm{kcal} \mathrm{mol}^{-1}$ for 2-phenyloxirane. This suggests that these oxidation products of gold-bound ethynylbenzene will remain bound to the surface. In our previous computational study of ethynylbenzene adsorption on $\mathrm{Au}(111)$ we showed that 
ethynylbenzene can adsorb directly to the surface by opening of the $\mathrm{C}-\mathrm{C}$ triple bond, and that the overall energy for this reaction is favourable. ${ }^{10}$ Here we have shown that if the reaction continues and ethynylbenzene is oxidized to one of the products shown in Figure 5, it will remain bound to the surface. The experimental results presented in this study support this assertion.

Experiments with 1,4-diethynylbenzene and gold nanoparticles. We recently showed that the $\alpha, \omega$ dithiol, 1,4-phenylenedimethanethiol, exhibits complex behaviour upon binding to gold surfaces, ${ }^{31}$ and that the functionalized surfaces are capable of binding gold nanoparticles from solution. In this work, we used the "double-ended" alkyne, 1,4-diethynylbenzene (Figure 1) to prepare surface structures under similar conditions to those used with ethynylbenzene, and subsequently exposed these surfaces to solutions of gold nanoparticles.

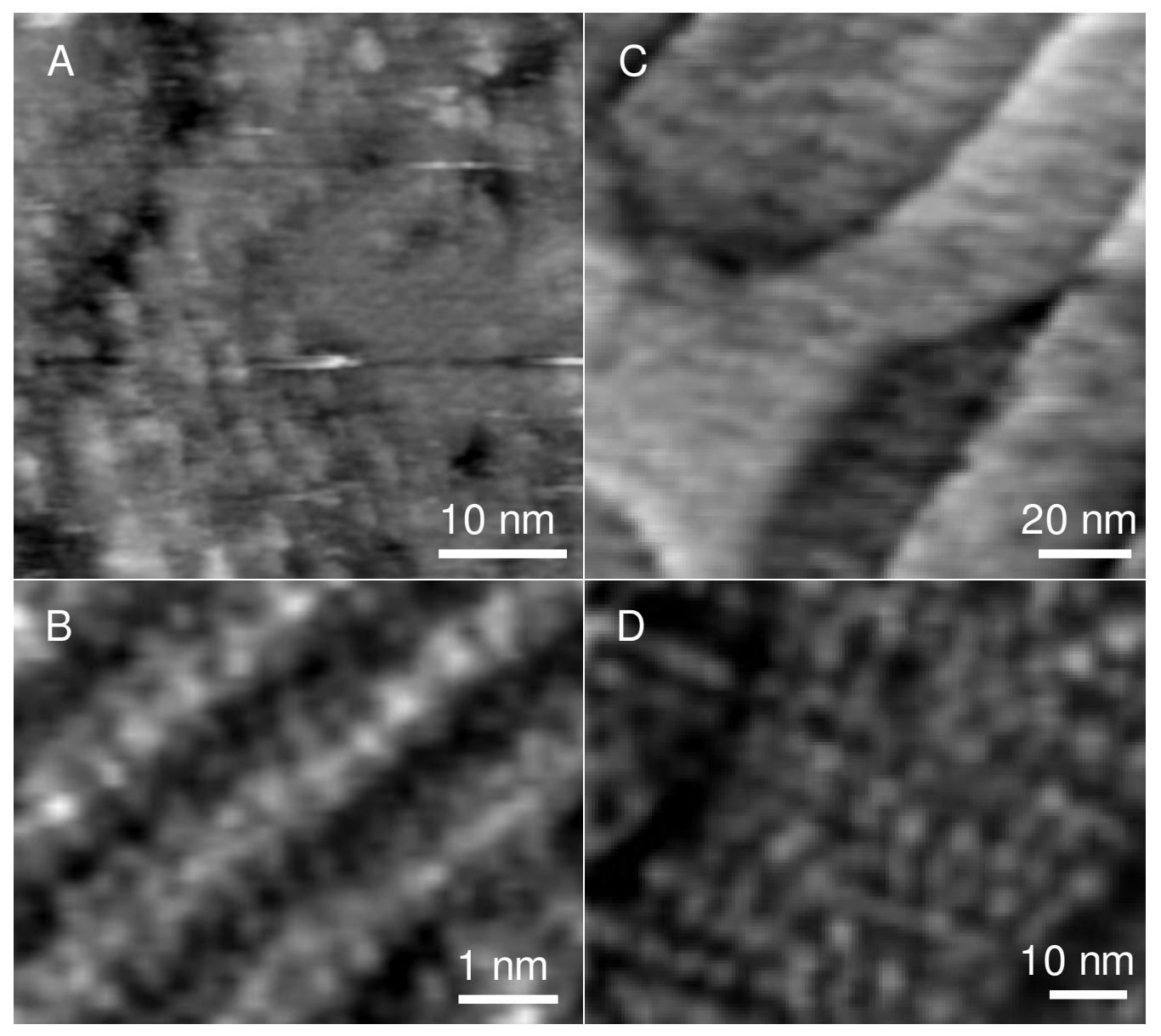

Figure 6. A: STM image of a Au(111) surface after immersion in a dichloromethane solution of 1,4diethynylbenzene and ammonium hydroxide. B: Magnified view of the order region from image A. C: 
Sample prepared using the same method as that shown in image A and then immerse in an aqueous solution of $5 \mathrm{~nm}$ gold particles. D: Magnified view of a region from image C.

Figure 6 shows scanning tunneling microscope (STM) images of the prepared surfaces. Figure 6A shows an STM image of a $\mathrm{Au}(111)$ surface after immersion in a dichloromethane solution of 1,4diethynylbenzene and ammonium hydroxide. Distinct ordered and disordered regions are visible. Figure 5B shows a magnified view of the well-ordered region shown in Figure 6A. Rows of ordered molecules are clearly visible with an inter-row spacing of $1.2 \mathrm{~nm}$. This data suggests that the molecules are oriented close to horizontally with respect to the gold surface, in a similar fashion to that indicated by the structures prepared from ethynylbenzene solutions.

Figure 6C shows an STM image of a sample prepared by immersing a 1,4-diethynylbenzene monolayer on $\mathrm{Au}(111)$ in an aqueous solution of $5 \mathrm{~nm}$ gold particles followed by thorough rinsing with solvent. The particles form a dense coverage on the surface. In the magnified view, Figure 6D, individual nanoparticles are clearly visible. The dense coverage shown in this image does not occur in all regions of the surface with other areas showing a sparser coverage of nanoparticles. From the areas surveyed, we estimate approximately $30 \%$ of the surface area is covered by the densely packed structure. Importantly, nanoparticle structures were not observed on samples prepared by immersion of bare gold substrates, or substrates prepared using ethynylbenzene, into nanoparticle solutions followed by rinsing. This indicates that the reaction that facilitates the binding of the terminal alkyne to the surface occurs at only one of the two $\mathrm{C} \equiv \mathrm{CH}$ units, so that one alkynyl group remains available to subsequently bind to the gold particles. We believe that this is the first example of ordered gold nanoparticle structures bound to a gold surface using an anchoring layer derived from a hydrocarbon.

\section{Conclusion}

We conclude that exposure of $\mathrm{Au}(111)$ surfaces to ethynylbenzene in basic ethanolic solution leads to the formation of a bound hydrocarbon monolayer that is not due to adventitious carbon. STM data show that a chemisorption process takes place (evidenced by etch pits) and that molecular-sized features are 
evident on the surface. XPS data are consistent with a layer that is not due to adventitious hydrocarbons but consists of oxygen-containing carbon species. Furthermore, the layer remains unchanged after one week under ambient conditions. ${ }^{1} \mathrm{H}$ NMR experiments show that ethynylbenzene does not oxidize under the conditions used to form the surface-bound layers but may be deprotonated in the basic environment. The oxidation of ethynylbenzene is therefore dependent on the gold surface. These findings show that planar gold surfaces can facilitate the oxidation of alkynes without the need for pre-dosing the surface with atomic oxygen. Calculations show that ethynylbenzene and its oxidation products phenylacetic acid and phenyloxirane have positive binding energies to the surface, and are therefore likely to remain bound to the gold albeit in a structure with no long-range organization. We suspect that incorporation of long alkyl chains in the 4-position of the aromatic ring of ethynylbenzene may lead to more crystalline structures. 1,4-diethynylbenzene also binds to $\mathrm{Au}(111)$ under similar conditions and can anchor gold nanoparticles deposited from solution to form dense, semi-regular arrays.

\section{Experimental}

Ethynylbenzene $^{32}$ was purchased from Aldrich and distilled before use. 1,4-Diethynylbenzene was prepared by literature procedure. ${ }^{33}$ Gold nanoparticles $\left(5 \mathrm{~nm}\right.$ diameter, $0.01 \%$ as $\mathrm{HAuCl}_{4}$ ) were purchased from Aldrich and used as received. Au(111) films on mica were purchased from Molecular Imaging, USA, and flame annealed before use.

Monolayer structures were prepared by immersing a gold substrate in a $1 \mathrm{mM}$ ethanolic solution of ethynylbenzene, or a $1 \mathrm{mM}$ dichloromethane solution of 1,4-diethynylbenzene with addition of ammonium hydroxide ( $1 \mu \mathrm{L}$ per $\mathrm{mL}$ of solvent). The container was sealed and placed in an oven at $60{ }^{\circ} \mathrm{C}$ for $24 \mathrm{~h}$. After cooling to room temperature, the sample was washed thoroughly with solvent and dried using a stream of $\mathrm{N}_{2}$ gas. In experiments using gold nanoparticles, structures prepared by the above methods were immersed in an aqueous solution of gold nanoparticles at room temperature for $24 \mathrm{~h}$ and then thoroughly rinsed with ethanol and dried using $\mathrm{N}_{2}$ gas.

Ellipsometry experiments were performed using a custom built rotating analyzer ellipsometer, incorporating a xenon lamp and monochromator at an angle of $70^{\circ}$ with respect to the surface normal. ${ }^{34}$ 
The wavelength was varied between $300 \mathrm{~nm}$ and $800 \mathrm{~nm}$, at $5 \mathrm{~nm}$ intervals. The freshly deposited gold surfaces were analyzed in order to determine each sample's baseline optical constants, $\Delta$ and $\Psi$, which describe the change in the polarization state of light on being reflected from the sample surface. After incubation and washing, $\Delta$ and $\Psi$ were measure again. The ellipsometry data were fitted in WVASE (J.A. Wollam Co. Inc., USA), and a comparison of the pre- and post-incubation results provided a measure of the SAMs thicknesses. A refractive index 1.55 (as stated by the manufacturer for ethynylbenzene) was assumed for an isotropic organic layer on the gold substrate.

Water contact angles were measured on freshly prepared samples using a Rame-Hart 100 goniometer. Advancing angles were measured immediately after placing a drop of water on the substrate with a micropipet.

XPS data were acquired using a ESCALAB220i-XL X-ray Photoelectron Spectrometer. The incident radiation was monochromatic $\mathrm{Al} \mathrm{K}$-alpha X-rays at $240 \mathrm{~W}(10 \mathrm{kV}, 24 \mathrm{~mA})$ with a spot size of $1 \mathrm{~mm}$ diameter. Survey (wide) scans were taken at an analyzer pass energy of $100 \mathrm{eV}$ and narrow high resolution scans at $20 \mathrm{eV}$.

STM images were acquired using a Nanosurf EasyScan system under ambient conditions. STM piezoelectric scanners were calibrated laterally, with graphite(0001) and Au(111), and vertically, using the height of the Au(111) steps $(0.22 \mathrm{~nm})$. The STM tip was prepared from Pt/Ir wire cut under ambient conditions. All images were acquired in a constant-current mode. Typical imaging conditions are bias voltages of \pm 0.2 to $\pm 1 \mathrm{~V}$ and a tunneling current of $3 \mathrm{pA}$ to $1 \mathrm{nA}$.

Polarisation Modulation Infrared Reflection Absorption Spectroscopy (PM-IRRAS) spectra were recorded on a Thermo Nicolet Nexus 870 FT-IR spectrometer in the PEM module, configured at a typical reflection angle of $83^{\circ}$ for samples with metallic substrates. Molecular structures were prepared using the procedure described above on gold surfaces freshly prepared by evaporative deposition. The PEM modulation frequency was centred at $1600 \mathrm{~cm}^{-1}$. The spectra were collected with a resolution of 4 $\mathrm{cm}^{-1}$ over 1000 scans. A background of an untreated gold-coated silicon wafer was collected under the same conditions. 
Computational studies employed the SIESTA methodology and software. ${ }^{35,36}$ This program implements linear-scaling density functional theory within periodic boundary conditions and is based on the linear combination of atomic orbitals approximation. A 4 x 4 unit cell, 4 layers thick was used to represent the gold surface. This unit cell is sufficiently large that there is negliable interaction between the periodic images of the adsorbed molecule. The valence electrons are described by atom-centered basis sets, and the nucleus/core electrons are represented by norm-conserving pseudopotentials. The key feature of this methodology is that the orbitals are strictly localized in real-space, with a cut-off radius defined by a single energy shift parameter for all atoms that represents the energy increase in the orbitals due to confinement. Exploitation of this locality leads directly to linear-scaling without the requirement of neglecting integrals based on a threshold value. We have previously investigated the convergence properties of the calculated energies against the various computational parameters, ${ }^{10}$ in the present work the interaction energies are converged to about $7 \mathrm{kcal} / \mathrm{mol}$. This figure does not include basis set effects, here we use double-zeta plus single polarization functions to represent all valence electrons.

${ }^{1} \mathrm{H}$ NMR spectra were recorded in D6-EtOD using a Bruker Avance 300 spectrometer at $300 \mathrm{MHz}$. The spectra were referenced internally to residual protic solvent ( $\delta 1.11$, methyl protons). Spectra were recorded of a $1 \mathrm{mM}$ solution of ethynylbenzene in EtOD $(1 \mathrm{~mL})$ before and after addition of ammonium hydroxide (ACS grade, Aldrich, 2 molar equivalents), and after incubation of the solution at $60{ }^{\circ} \mathrm{C}$ for 42 hours in a sealed NMR tube.

Acknowledgment We thank Mr Steve Moody for assistance with ellipsometry measurements and Dr Bill Gong, UNSW, for XPS experiments. AMM holds an Australian Research Council Post-doctoral Research Fellowship.

Supporting Information Available. XPS and ${ }^{1} \mathrm{H}$ NMR spectra and computational details.

\section{References}

1. (a) Schreiber, F. Prog. Surf. Sci. 2000, 65, 151. (b) Ulman, A. Chem. Rev. 1996, 96, 1533. 
2. Jurkiewicz-Herbich, M.; Slojkowska, R.; Zawada, K.; Bukowska, J. Electrochim. Acta. 2002, 47, 2429.

3. Dirk, S. M.; Tour, J. M. Tetrahedron, 2003, 59, 287.

4. (a) Chen, J.; Calvet, L. C.; Reed, M. A.; Carr, D. W.; Grubisha, D. S.; Bennett, D. W. Chem. Phys. Lett. 1999, 313, 741. (b) Lin, S.; McCarley, R. L. Langmuir, 1999, 15, 151. (c) Horswell, S. L.; O’Neil, I. A.; Schiffrin, D. J. J. Phys. Chem. B. 2001, 105, 941.

5. DuBose, D. L.; Robinson, R. E.; Holovics, T. C.; Moody, D. R.; Weintrob, E. C.; Berrie, C. L.; Barybin, M. V. Langmuir 2006, 22, 4599.

6. Tour, J. M. Acc. Chem. Res. 2000, 33, 791.

7. Cross, R. J.; Davidson, M. F.; McLennan, A. J. J. Organomet. Chem. 1984, 265, C37.

8. (a) Feilchenfeld, H.; Weaver, M. J. J. Phys. Chem. 1989, 93, 4276. (b) Patterson, M. L.; Weaver, M. J. J. Phys. Chem. 1985, 89, 5046.

9. Joo, S-W.; Kim, K. J. Raman Spectroscopy 2004, 35, 549.

10. Ford, M. J.; Hoft, R. C.; McDonagh, A. J. Phys. Chem. B 2005, 109, 20387.

11. Deng, X.; Friend, C. M. J. Am. Chem. Soc. 2005, 127, 17178.

12. Pritzkow, W.; Rao, T. S. S. J. Prakt. Chem. 1985, 327, 887.

13. Rao, T. S. S.; Awasthi, S. J. Indian Chem. Soc. 2003, 80, 1129.

14. Tao, Y-T.; Wu, C-C.; Eu, J-Y.; Lin, W-L.; Wu, K-C.; Chen, C-h. Langmuir 1997, 13, 4018.

15. Noh, J.; Hara, M. Langmuir 2001, 17, 7280.

16. Repain, V.; Berroir, J. M.; Rousset, S.; Lecoeur, J. Europhys. Lett. 1999, 47, 435. 
17. Poirier, G.E. Langmuir 1997, 13, 2019.

18. Yang, G.; Liu, G-y. J. Phys. Chem. B 2003, 107, 8746.

19. Iucci, G.; Carravetta, V.; Altamura, P.; Russo, M. V.; Paolucci, G.; Goldoni, A.; Polzonetti, G. Chem. Phys. 2004, 302, 43.

20. Whelan, C. M.; Ghijsen, J.; Pireaux, J-J.; Maex, K. Thin Solid Films 2004, 464, 388.

21. Piao, H.; Enever, M. C. N.; Adib, K.; Hrbek, J.; Barteau, M. A. Surf. Sci. 2004, 571, 139.

22. Iucci, G.; Carravetta, V.; Paolucci, G.; Goldoni, A.; Russo, M. V.; Polzonetti, G. Chem. Phys. 2005, $310,43$.

23. Klust, A.; Madix, R. J.; J. Am. Chem. Soc. 2006, 128, 1034.

24. Bain, C. D.; Troughton, E. B.; Tao, Y-T.; Evall, J.; Whitesides, G. M.; Nuzzo, R. G. J. Am. Chem. Soc. 1989, 111, 321.

25. Stoycheva, S.; Himmelhaus, M.; Fick, J.; Kornviakov, A.; Grunze, M.; Ulman, A. Langmuir 2006, 22, 4170.

26. Mateo-Martí, E; Briones, C,Román, E.; Briand, E.; Pradier, C. M.; Martín-Gago, J. A. Langmuir 2005, 21, 9510.

27. Ordejon, P.; Artacho, E.; Soler, J. M. Phys. Rev. B (Rapid Comm.), 1996, 53, R10441

28. Soler, J. M.; Artacho, E.; Gale, J.; Garcia, A.; Junquera, J.; Ordejon, P.; Sanchez-Portal, D. J. Phys.: Condens. Matter, 2002, 14, 2745

29. R.C. Hoft, J.D. Gale, and M.J. Ford, Mol. Sim. 32(8) 595-600 (2006)).

30. Boys, S. B.; Bernardi, F. Mol. Phys. 1970, 19, 553. 
31. Zareie, H. M.; McDonagh, A. M.; Edgar, J.; Ford, M. J.; Cortie, M. B.; Phillips, M. R. Chem. Mater. 2006, 18, 2376.

32. In this article we use the IUPAC nomenclature "ethynylbenzene". The compound was purchased using the common name "phenylacetylene".

33. Takahashi, S.; Kuroyama, Y.; Sonogashira, K.; Hagihara, N. Synthesis 1980, 8, 627.

34. Bendavid, A.; Burns, C. J.; Field, L. D.; Hashimoto, K.; Ridley, D. D.; Sandanayake, K. R. A. S.; Wieczorek, L. J. Org. Chem. 2001, 66, 3709.

35. Ordejon, P.; Artacho, E.; Soler, J. M. Phys. Rev. B: Condens. Matter 1996, 53, R10441.

36. Soler, J. M.; Artacho, E.; Gale, J. D.; Garcia, A.; Junquera, J.; Ordejon, P.; Sánchez-Portal, D. J. Phys.: Condens. Matter 2002, 14, 2745.

\section{Table of Contents Graphic.}



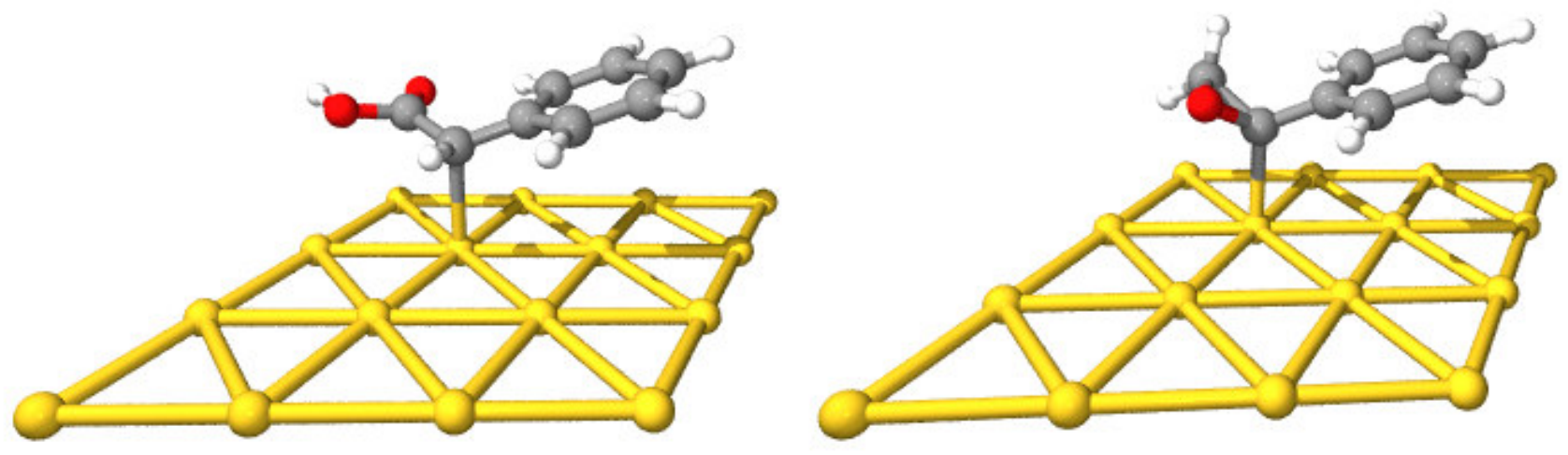

Oxidation products of ethynylbenzene on gold(111) surfaces. 